\title{
UPPER GASTROINTESTINAL BLEEDING; ENDOSCOPIC FINDINGS IN PATIENTS
}

2. MBBS, FCPS (Med) Assistant Professor of Medicine Independent Medical College, Independent University Hospital Faisalabad

3. MBBS, FCPS (Med) Associate Professor of Medicine Independent Medical College, Independent University Hospital, Faisalabad

\section{Correspondence Address:}

Dr. Muhammad Zakria

Associate Professor of Medicine

Independent Medical College,

Independent University Hospital,

Faisalabad

zakriadr@yahoo.com

sarfraz661@hotmail.com

Article received on: 02/12/2016

Accepted for publication: 20/01/2017

Received after proof reading: 14/02/2017

\section{Dr. Mughees Ather ${ }^{1}$, Dr. Muhammad Sarfraz ${ }^{2}$, Dr. Muhammad Zakria ${ }^{3}$}

ABSTRACT... Background: Upper gastrointestinal bleeding (UGIB) is a common medical condition requiring mostly hospitalization and resuscitation. Patients with upper Gl bleeding have high morbidity. The investigation of choice in upper Gl bleeding is upper Gl endoscopy, because endoscopy has good, the complication rate with endoscopy are low, another advantage of using endoscopy is therapeutic interventions as well. The causes of upper GI bleeding are numerous most frequent causes being in decreasing order of frequency peptic ulcer, esophageal varices, mallory-weis tears. Other causes include tumors, erosions and arterio-venous malformations. ${ }^{3}$ Objectives: To determine the endoscopic findings in patients presenting with UGIB and frequency of various findings among these patients according to gender and age in medical ward of Allied Hospital Faisalabad. Study Design: Retrospectively reviewed and analyzed. Setting: Medical Unit 2 of Allied Hospital Faisalabad. Period: 06 month between January 2015 to June 2015. Methods: The record of 120 patients who underwent endoscopy for upper gastrointestinal bleeding. Results: Data was collected and entered on SPSS. Statistical data analysis was performed with chi-square. Statistical significance was determined at $P<0.05$. Information obtained from upper gastrointestinal endoscopy were then recorded according to age, gender and presented in form of tables. Conclusions: It has been found that esophageal varices was the most commonest cause of upper gastrointestinal bleeding in the study probably due to the high prevalence of hepatitis infection and chronic liver disease in our population.

Key words: $\quad$ Upper gastrointestinal bleeding (UGIB), Esophageal varices, Fundal varices.

Article Citation: Ather M, Sarfraz M, Zakria M. Upper gastrointestinal bleeding; endoscopic findings in patients. Professional Med J 2017;24(2):335-341.

DOI: 10.17957/TPMJ/17.3711

\section{INTRODUCTION}

Upper gastrointestinal bleeding (UGIB) is a common emergency medical condition. The bleeding anywhere from esophagus to the small intestine where ligament of treitz attached will be presented as upper gastrointestinal bleeding and patient with UGIB may have complaints of haematemesis and melena or having complaint of only melena if the bleeding is not severe. Occasionally in massive UGIB fresh blood may appear in stool. Most of these patients need admission, initial emergency resuscitative measures are taken like maintaining airways, breathing, fluid and blood transfusion. If bleeding is severe emergency gastro-esophagoscopy along with resuscitative measures are taken simultaneously, while in mild to moderate bleeding cases initially patient is stabilized then upper Gl endoscopy is done. UGIB has high mortality and morbidity if timely appropriate treatment measures are not taken. ${ }^{1}$ Bleeding from the upper gastro intestinal tract is approximately 4 times as common as bleeding from the lower gastro intestinal tract with mortality rates from UGIB are $6-10 \%$ overall. $^{2}$

Therearea lot of diverse diseases causing bleeding from upper gastrointestinal tract including peptic ulcer, erosive gastritis, esophageal varices, bleeding from esophageal tears due to excessive vomiting, gastric carcinoma, esophageal carcinoma and various bleeding disorders. In western population the most common culprit lesion causing bleeding from UGIT is peptic ulcer $(50 \%)$ followed by esophageal varices $(14 \%) .{ }^{3}$ Timely taken resuscitative and therapeutic 
measures will reduce mortality and morbidity in these patients. ${ }^{4,5}$

The investigation and treatment modality of choice is upper gastrointestinal endoscopy because of its affordability and accuracy in picking up various lesions. ${ }^{3,6}$ This study was carried out to evaluate the endoscopic findings in patients presenting with UGIB and its frequency among these patients according to gender and age.

\section{METHOD}

The record of patients presented with bleeding from UGIT in medical unit 2 of Allied hospital between Jan 2015 to June 2015 was reviewed and analyzed retrospectively. The record of all patients (120 patients) presented with UGIB and underwent upper Gl endoscopy was reviewed. The patients who had repeat endoscopy were excluded.

Data that was collected from endoscopy record of the hospital include age sex, history of upper gastrointestinal bleeding and documentation of endoscopic findings in the endoscopy register. All patients were resuscitated and stabilized hermodynamically before endoscopy was done.

Data was collected using questionnaires and entered on SPSS. Statistical data analysis was performed with chi-square. Statistical significance was determined at $P<0.05$. Information obtained from upper Gl endoscopy were then recorded according to age, gender and presented in form of tables.

The study was approved by the Ethical Research Committee of allied hospital.

\section{RESULTS}

A total of 120 patients underwent upper gastrointestinal endoscopy during the 6 month period covered in the study between January 2015 to June 2015. Out of the total number of 120 patients 70 patients were male $(58.3 \%)$, while females were 50 in number (41.7\%), and the mean age was 42 years \pm SD (standard deviation) 15.88. The male to female ratio was $1.4: 1$.
As for as age distribution of UGIB is concerned, most number of patient were is the age group of 41-60 years. 79 Patients were in the age group of 41-60 years who presented with UGIB. The most frequent cause of UGIB is esophageal varices in our study. Out of 79, 61 patient had esophageal varices.

Esophageal varices was also the most frequent cause in female patients in our study (84\%). In male patient esophageal varices is found to be a cause of UGIB in $72.9 \%$ of the male population in our study.

The freqnecy of various cause of UGIB in our study in order of decreasing frequencies are esophageal varices (77.5\%), Gastric ulcer / Gastritis (6.7\%), Fundal varices (5.8\%), Esophagitis / Esophageal ulcer (2.5\%)

\section{Analysis}

\begin{tabular}{|c|c|c|c|c|c|}
\hline \multicolumn{3}{|c|}{ Classes } & Frequency & \multicolumn{2}{|r|}{ Percent } \\
\hline \multicolumn{3}{|c|}{$1-20$} & 3 & & 2.5 \\
\hline \multicolumn{3}{|c|}{$21-40$} & 17 & & 14.2 \\
\hline \multicolumn{3}{|c|}{$41-60$} & 79 & & 65.8 \\
\hline \multicolumn{3}{|c|}{$61-80$} & 18 & & 15.0 \\
\hline \multicolumn{3}{|c|}{$81-100$} & 3 & & 2.5 \\
\hline \multicolumn{3}{|c|}{ Total } & 120 & & 100.0 \\
\hline \multicolumn{6}{|c|}{ Descriptive Statistics } \\
\hline & $\mathrm{N}$ & Minimum & Maximum & Mean & Std. Deviation \\
\hline age & 120 & 10 & 95 & 50.83 & 13.580 \\
\hline
\end{tabular}

\begin{tabular}{|c|c|c|}
\hline Gender & Frequency & Percent \\
\hline Female & 50 & 41.7 \\
\hline Male & 70 & 58.3 \\
\hline Total & 120 & 100.0 \\
\hline \multicolumn{2}{|c|}{ Table-1.2. Gender Distribution } \\
\hline
\end{tabular}

\begin{tabular}{|l|c|c|}
\hline Esophageal Varices & Frequency & Percent \\
\hline Negative & 27 & 22.5 \\
\hline Positive & 93 & 77.5 \\
\hline Total & 120 & 100.0 \\
\hline \multicolumn{2}{|l}{ Table-1.3. Esophageal Varices Distribution } \\
\hline
\end{tabular}




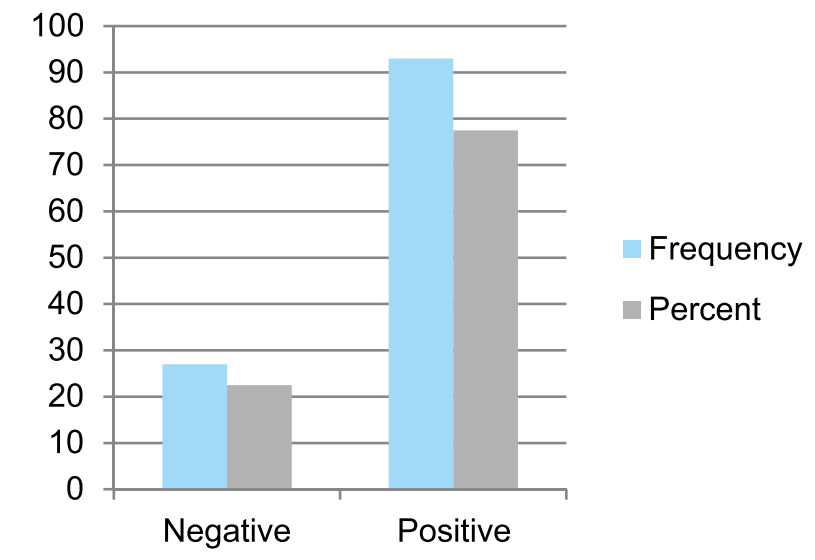

\begin{tabular}{|l|c|c|}
\hline $\begin{array}{c}\text { Esophageal Ulcer / } \\
\text { Eesohagtis }\end{array}$ & Frequency & Percent \\
\hline Negative & 117 & 97.5 \\
\hline Positive & 3 & 2.5 \\
\hline Total & 120 & 100.0 \\
\hline
\end{tabular}

Table-1.4. Esophageal Ulcer / Eesophagtis Distribution

\begin{tabular}{|c|c|c|}
\hline Gestric Ulcer / Gastritis & Frequency & Percent \\
\hline Negative & 112 & 93.3 \\
\hline Positive & 8 & 6.7 \\
\hline Total & 120 & 100.0 \\
\hline
\end{tabular}

Table-1.5. Gastric Ulcer / Gastritis Distribution

\begin{tabular}{|c|c|c|}
\hline Fundal Varices & Frequency & Percent \\
\hline Negative & 113 & 94.2 \\
\hline Positive & 7 & 5.8 \\
\hline Total & 120 & 100.0 \\
\hline
\end{tabular}

Table-1.6. Fundal Varices Distribution

\begin{tabular}{|c|c|c|}
\hline Duodenal Ulcer & Frequency & Percent \\
\hline Negative & 120 & 100.0 \\
\hline
\end{tabular}

Table-1.7. Duodenal Ulcer Distribution

Table-I. Distribution of patients with UGIB according to age.

\begin{tabular}{|c|c|c|c|}
\hline \multirow{2}{*}{ Age } & \multicolumn{2}{|c|}{ Esophageal_Varices } & \multirow{2}{*}{ Total } \\
\cline { 2 - 4 } & Negative & Positive & \\
\hline $1-20$ & $1(33.3 \%)$ & $2(66.7 \%)$ & $3(100.0 \%)$ \\
\hline $21-40$ & $4(23.5 \%)$ & $13(76.5 \%)$ & $17(100.0 \%)$ \\
\hline $41-60$ & $18(22.8 \%)$ & $61(77.2 \%)$ & $79(100.0 \%)$ \\
\hline $61-80$ & $3(16.7 \%)$ & $15(83.3 \%)$ & $18(100.0 \%)$ \\
\hline $81-100$ & $1(33.3 \%)$ & $2(66.7 \%)$ & $3(100.0 \%)$ \\
\hline Total & $27(22.5 \%)$ & $93(77.5 \%)$ & $120(100.0 \%)$ \\
\hline
\end{tabular}

\begin{tabular}{|c|c|c|c|c|}
\hline \multicolumn{5}{|c|}{ Chi-Square Tests } \\
\hline & & Value & Df & $\begin{array}{l}\text { Asymp. Sig. } \\
\text { (2-sided) }\end{array}$ \\
\hline \multicolumn{2}{|c|}{ Pearson Chi-Square } & $.769^{a}$ & 4 & .943 \\
\hline \multicolumn{2}{|c|}{ Likelihood Ratio } & .758 & 4 & .944 \\
\hline \multicolumn{2}{|c|}{$\mathrm{N}$ of Valid Cases } & \multicolumn{2}{|c|}{120} & \\
\hline \multicolumn{5}{|c|}{ Table-2.1. Esophageal Varices according to age } \\
\hline & \multicolumn{3}{|c|}{ Crosstab } & \\
\hline & \multicolumn{3}{|c|}{$\begin{array}{c}\text { Esophageal Ulcer / } \\
\text { Eesohagtis }\end{array}$} & \\
\hline Age & \multicolumn{2}{|c|}{ Negative } & Positive & Total \\
\hline $1-20$ & \multicolumn{2}{|c|}{$3(100.0 \%)$} & $0(.0 \%)$ & $3(100.0 \%)$ \\
\hline $21-40$ & \multicolumn{2}{|c|}{17 (100.0\%) } & $0(.0 \%)$ & $17(100.0 \%)$ \\
\hline $41-60$ & \multicolumn{2}{|c|}{77 (97.5\%) } & $2(2.5 \%)$ & $79(100.0 \%)$ \\
\hline $61-80$ & \multicolumn{2}{|c|}{$17(94.4 \%)$} & $1(5.6 \%)$ & $18(100.0 \%)$ \\
\hline $81-100$ & \multicolumn{2}{|c|}{$3(100.0 \%)$} & $0(.0 \%)$ & $3(100.0 \%)$ \\
\hline \multirow{2}{*}{ Total } & \multicolumn{2}{|c|}{$117(97.5 \%)$} & $3(2.5 \%)$ & $120(100.0 \%)$ \\
\hline & \multicolumn{2}{|l|}{$97.5 \%$} & $2.5 \%$ & $100.0 \%$ \\
\hline
\end{tabular}

\begin{tabular}{|l|c|c|c|}
\hline \multicolumn{4}{|c|}{ Chi-Square Tests } \\
\hline & Value & Df & $\begin{array}{c}\text { Asymp. Sig. } \\
\text { (2-sided) }\end{array}$ \\
\hline Pearson Chi-Square & $1.280^{\mathrm{a}}$ & 4 & .865 \\
\hline Likelihood Ratio & 1.679 & 4 & .794 \\
\hline N of Valid Cases & 120 & & \\
\hline Table-2.2. Age and Esophageal Ulcer / Eesophagtis \\
\hline
\end{tabular}

\begin{tabular}{|c|c|c|c|}
\hline \multirow{2}{*}{ Age } & \multicolumn{2}{|c|}{ Gastric Ulcer / Gastritis } & \multirow{2}{*}{ Total } \\
\hline & Negative & Positive & \\
\hline $1-20$ & $2(66.7 \%)$ & $1(33.3 \%)$ & $3(100.0 \%)$ \\
\hline $21-40$ & $16(94.1 \%)$ & $1(5.9 \%)$ & $17(100.0 \%)$ \\
\hline $41-60$ & 73 (92.4\%) & $6(7.6 \%)$ & $79(100.0 \%)$ \\
\hline $61-80$ & $18(100.0 \%)$ & $0(.0 \%)$ & $18(100.0 \%)$ \\
\hline $81-100$ & $3(100.0 \%)$ & $0(.0 \%)$ & $3(100.0 \%)$ \\
\hline Total & $112(93.3 \%)$ & $8(6.7 \%)$ & $120(100.0 \%)$ \\
\hline
\end{tabular}

\begin{tabular}{|l|c|c|c|}
\hline \multicolumn{4}{|c|}{ Chi-Square Tests } \\
& Value & df & $\begin{array}{c}\text { Asymp. Sig. } \\
\text { (2-sided) }\end{array}$ \\
\hline Pearson Chi-Square & $5.055^{\mathrm{a}}$ & 4 & .082 \\
\hline Likelihood Ratio & 4.893 & 4 & .298 \\
\hline $\begin{array}{c}\text { N of Valid Cases } \\
\text { Table-2.3. Age and Gastric Ulcer / Gastritis }\end{array}$
\end{tabular}




\begin{tabular}{|c|c|c|c|}
\hline \multirow{2}{*}{ Age } & \multicolumn{2}{|c|}{ Fundal Varices } & \multirow{2}{*}{ Total } \\
\cline { 2 - 4 } & Negative & Positive & \\
\hline $1-20$ & $3(100.0 \%)$ & $0(.0 \%)$ & $3(100.0 \%)$ \\
\hline $21-40$ & $15(88.2 \%)$ & $2(11.8 \%)$ & $17(100.0 \%)$ \\
\hline $41-60$ & $75(94.9 \%)$ & $4(5.1 \%)$ & $79(100.0 \%)$ \\
\hline $61-80$ & $18(100.0 \%)$ & $0(.0 \%)$ & $18(100.0 \%)$ \\
\hline $81-100$ & $2(66.7 \%)$ & $1(33.3 \%)$ & $3(100.0 \%)$ \\
\hline Total & $113(94.2 \%)$ & $7(5.8 \%)$ & $120(100.0 \%)$ \\
\hline
\end{tabular}

\begin{tabular}{|l|c|c|c|}
\hline \multicolumn{3}{|c|}{ Chi-Square Tests } \\
& Value & df & $\begin{array}{c}\text { Asymp. Sig. } \\
\text { (2-sided) }\end{array}$ \\
\hline Pearson Chi-Square & $6.605^{\mathrm{a}}$ & 4 & .058 \\
\hline Likelihood Ratio & 5.572 & 4 & .233 \\
\hline N of Valid Cases & 120 & & \\
\hline
\end{tabular}

Table-2.4. Age and Fundal Varices

\begin{tabular}{|l|c|c|}
\hline \multicolumn{1}{|c|}{ Age } & $\begin{array}{c}\text { Duodenal_Ulcer } \\
\text { Negative }\end{array}$ & Total \\
\hline $1-20$ & $3(100.0 \%)$ & $3(100.0 \%)$ \\
\hline $21-40$ & $17(100.0 \%)$ & $17(100.0 \%)$ \\
\hline $41-60$ & $79(100.0 \%)$ & $79(100.0 \%)$ \\
\hline $61-80$ & $18(100.0 \%)$ & $18(100.0 \%)$ \\
\hline $81-100$ & $3(100.0 \%)$ & $3(100.0 \%)$ \\
\hline Total & $120(100.0 \%)$ & $120(100.0 \%)$ \\
\hline
\end{tabular}

\begin{tabular}{|c|c|c|c|}
\hline \multicolumn{4}{|c|}{ Chi-Square Tests } \\
\hline & & \multicolumn{2}{|c|}{ Value } \\
\hline \multicolumn{2}{|c|}{ Pearson Chi-Square } & \multicolumn{2}{|c|}{.$^{a}$} \\
\hline \multicolumn{2}{|c|}{$\mathrm{N}$ of Valid Cases } & \multicolumn{2}{|c|}{120} \\
\hline \multicolumn{4}{|c|}{ Table-2.5. Age and Duodenal Ulcer } \\
\hline \multirow{2}{*}{ Gender } & \multicolumn{2}{|c|}{ Esophageal Varices } & \multirow{2}{*}{ Total } \\
\hline & Negative & Positive & \\
\hline Female & $8(16.0 \%)$ & $42(84.0 \%)$ & $50(100.0 \%)$ \\
\hline Male & $19(27.1 \%)$ & $51(72.9 \%)$ & $70(100.0 \%)$ \\
\hline Total & $27(22.5 \%)$ & $93(77.5 \%)$ & $120(100.0 \%)$ \\
\hline
\end{tabular}

\begin{tabular}{|c|c|c|c|c|c|}
\hline \multicolumn{6}{|c|}{ Chi-Square Tests } \\
\hline & Value & df & $\begin{array}{l}\text { Asymp. } \\
\text { Sig. } \\
\text { (2-sided) }\end{array}$ & $\begin{array}{c}\text { Exact } \\
\text { Sig. } \\
\text { (2-sided) }\end{array}$ & $\begin{array}{l}\text { Exact Sig. } \\
\text { (1-sided) }\end{array}$ \\
\hline $\begin{array}{l}\text { Pearson } \\
\text { Chi-Square }\end{array}$ & $2.077^{\mathrm{a}}$ & 1 & .150 & & \\
\hline $\begin{array}{l}\text { Continuity } \\
\text { Correction }^{\mathrm{b}}\end{array}$ & 1.487 & 1 & .223 & & \\
\hline $\begin{array}{l}\text { Likelihood } \\
\text { Ratio }\end{array}$ & 2.138 & 1 & .144 & & \\
\hline $\begin{array}{l}\text { Fisher's } \\
\text { Exact Test }\end{array}$ & & & & .186 & .111 \\
\hline $\begin{array}{l}\mathrm{N} \text { of Valid } \\
\text { Cases }^{\mathrm{b}}\end{array}$ & 120 & & & & \\
\hline
\end{tabular}

\begin{tabular}{|c|c|c|c|c|c|c|}
\hline \multicolumn{7}{|c|}{ Esophageal Ulcer / Eesophagtis } \\
\hline Gender & \multicolumn{2}{|c|}{ Negative } & \multicolumn{2}{|c|}{ Positive } & \multicolumn{2}{|r|}{ Total } \\
\hline Female & \multicolumn{2}{|c|}{$50(100.0 \%)$} & \multicolumn{2}{|c|}{$0(.0 \%)$} & \multicolumn{2}{|c|}{$50(100.0 \%)$} \\
\hline Male & \multicolumn{2}{|c|}{$67(95.7 \%)$} & \multicolumn{2}{|c|}{$3(4.3 \%)$} & \multicolumn{2}{|c|}{70 (100.0\%) } \\
\hline Total & \multicolumn{2}{|c|}{$117(97.5 \%)$} & \multicolumn{2}{|c|}{$3(2.5 \%)$} & \multicolumn{2}{|c|}{$120(100.0 \%)$} \\
\hline \multicolumn{7}{|c|}{ Chi-Square Tests } \\
\hline & Value & df & $\begin{array}{l}\text { Asymp. } \\
\text { Sig. } \\
\text { (2-sided) }\end{array}$ & \multicolumn{2}{|c|}{$\begin{array}{l}\text { Exact Sig. } \\
\text { (2-sided) }\end{array}$} & $\begin{array}{l}\text { Exact Sig. } \\
\text { (1-sided) }\end{array}$ \\
\hline $\begin{array}{l}\text { Pearson } \\
\text { Chi-Square }\end{array}$ & $2.198^{\mathrm{a}}$ & 1 & .138 & & & \\
\hline $\begin{array}{l}\text { Continuity } \\
\text { Correction }\end{array}$ & .791 & 1 & .374 & & & \\
\hline $\begin{array}{l}\text { Likelihood } \\
\text { Ratio }\end{array}$ & 3.289 & 1 & .070 & & & \\
\hline $\begin{array}{l}\text { Fisher's } \\
\text { Exact Test }\end{array}$ & & & & & 65 & .195 \\
\hline $\begin{array}{l}\mathrm{N} \text { of Valid } \\
\text { Cases }^{\mathrm{b}}\end{array}$ & 120 & & & & & \\
\hline \multicolumn{7}{|c|}{ Table-3.2. Gender and Esophageal Ulcer / Eesophagtis } \\
\hline & \multicolumn{4}{|c|}{ Gastric Ulcer / Gastritis } & \multirow{2}{*}{\multicolumn{2}{|c|}{ Tata }} \\
\hline Gender & \multicolumn{2}{|c|}{ Negative } & \multicolumn{2}{|c|}{ Positive } & & \\
\hline Female & \multicolumn{2}{|c|}{$47(94.0 \%)$} & \multicolumn{2}{|c|}{$3(6.0 \%)$} & \multicolumn{2}{|c|}{$50(100.0 \%)$} \\
\hline Male & \multicolumn{2}{|c|}{$65(92.9 \%)$} & \multicolumn{2}{|c|}{$5(7.1 \%)$} & \multicolumn{2}{|c|}{70 (100.0\%) } \\
\hline Total & \multicolumn{2}{|c|}{112 (93.3\%) } & \multicolumn{2}{|c|}{8 (6.7\%) } & \multicolumn{2}{|c|}{$120(100.0 \%)$} \\
\hline
\end{tabular}

\section{Chi-Square Tests}

\begin{tabular}{|c|c|c|c|c|c|c|}
\hline & Value & df & $\begin{array}{l}\text { Asymp. } \\
\text { Sig. } \\
\text { (2-sided) }\end{array}$ & \multicolumn{2}{|c|}{$\begin{array}{l}\text { Exact Sig. } \\
\text { (2-sided) }\end{array}$} & $\begin{array}{l}\text { Exact Sig. } \\
\text { (1-sided) }\end{array}$ \\
\hline $\begin{array}{l}\text { Pearson } \\
\text { Chi-Square }\end{array}$ & $.061^{\mathrm{a}}$ & 1 & .805 & & & \\
\hline $\begin{array}{l}\text { Continuity } \\
\text { Correction }^{\mathrm{b}}\end{array}$ & .000 & 1 & 1.000 & & & \\
\hline $\begin{array}{l}\text { Likelihood } \\
\text { Ratio }\end{array}$ & .062 & 1 & .804 & & & \\
\hline $\begin{array}{l}\text { Fisher's } \\
\text { Exact Test }\end{array}$ & & & & $1 . \mathrm{C}$ & & .556 \\
\hline $\begin{array}{l}\mathrm{N} \text { of Valid } \\
\text { Cases }^{\mathrm{b}}\end{array}$ & 120 & & & & & \\
\hline \multicolumn{7}{|c|}{ Table-3.3. Gender and Gastric Ulcer / Gastritis } \\
\hline & \multicolumn{4}{|c|}{ Fundal_Varcies } & \multirow{2}{*}{\multicolumn{2}{|c|}{ Total }} \\
\hline Gender & \multicolumn{2}{|c|}{ Negative } & \multicolumn{2}{|c|}{ Positive } & & \\
\hline Female & \multicolumn{2}{|c|}{$45(90.0 \%)$} & \multicolumn{2}{|c|}{$5(10.0 \%)$} & \multicolumn{2}{|c|}{$50(100.0 \%)$} \\
\hline Male & \multicolumn{2}{|c|}{68 (97.1\%) } & \multicolumn{2}{|c|}{2 (2.9\%) } & \multicolumn{2}{|c|}{70 (100.0\%) } \\
\hline Total & \multicolumn{2}{|c|}{113 (94.2\%) } & \multicolumn{2}{|c|}{7 (5.8\%) } & \multicolumn{2}{|c|}{120 (100.0\%) } \\
\hline
\end{tabular}

\section{Chi-Square Tests}




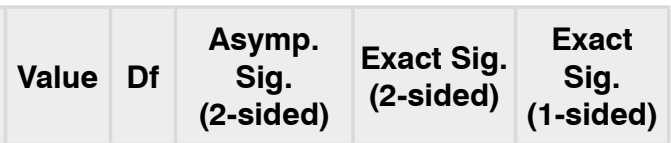

\begin{tabular}{|l|l|l|l|l|l|}
\hline $\begin{array}{l}\text { Pearson } \\
\text { Chi-Square }\end{array}$ & $2.709^{\mathrm{a}}$ & 1 & .100 & \\
\hline $\begin{array}{l}\text { Continuity } \\
\text { Correction }\end{array}$ & 1.565 & 1 & .211 & \\
\hline $\begin{array}{l}\text { Likelihood } \\
\text { Ratio }\end{array}$ & 2.694 & 1 & .101 & \\
\hline
\end{tabular}

\begin{tabular}{|l|l|l|l|l|}
\hline \begin{tabular}{l|l|l|} 
Fisher's \\
Exact Test
\end{tabular} & & & .127 & .106 \\
\hline $\begin{array}{l}\text { N of Valid } \\
\text { Cases }^{b}\end{array}$ & 120 & & & \\
\hline
\end{tabular}

Table-3.4. Gender and Fundal Varices

\begin{tabular}{|c|c|c|}
\hline \multicolumn{3}{|c|}{ Crosstab } \\
\hline & $\begin{array}{c}\text { Duodenal Ulcer } \\
\text { Negative }\end{array}$ & Total \\
\hline Gender & $50(100.0 \%)$ & $50(100.0 \%)$ \\
\hline Female & $70(100.0 \%)$ & $70(100.0 \%)$ \\
\hline Male & $120(100.0 \%)$ & $120(100.0 \%)$ \\
\hline Total & & \\
\hline
\end{tabular}

\section{Chi-Square Tests}

\begin{tabular}{|c|c|}
\hline & Value \\
\hline $\mathrm{N}$ of Valid Cases &.$^{\mathrm{a}}$ \\
\hline
\end{tabular}

Table-3.5. Gender and Duodenal Ulcer

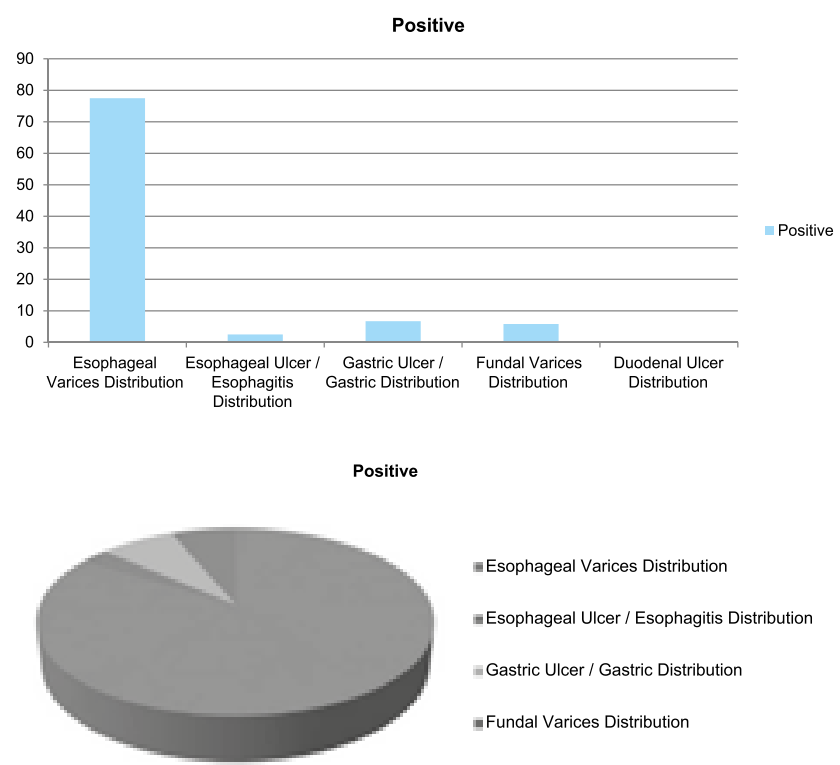

\section{DISCUSSION}

In emergency we mostly come across patients with haematemesis due to bleeding from esophagus, stomach or duodenum by variety of diseases, a part from managing the patient on priority basis as the condition demands it is vital to perform oesophago-gastroscopy as early as possible to find the cause and performing appropriate therapeutic intervention as well to decrease morbidity and mortality. The procedure to do endoscopy is simple. Patient is asked not to take anything for $6-8$ hours before procedure. Therapeutic modalities includes band ligation of bleeding vessel, injecting sclerosing agent, radio-frequency ablation etc. The most common causes of upper Gl bleeding are esophageal varices and peptic ulcer among other causes mentioned above.

In this study it was found that mean age of the patients was 50.83 years \pm Standard deviation 13.580. The mean age and standard deviation in this study is higher than those of other studies conducted in Africa where the mean age was 42.9years \pm SD 15.88.7,8 Male to female ratio found to be 1.4:1. Male patients were 70 and female patients were 50 in number.

Most of the patients in our study were in the age group of 40-60 years. Esophageal varices was the most common lesion found overall $(77.5 \%)$. it was also the most commonest lesion in $84 \%$ of females slightly more as compared to male patients $(72.9 \%)$. The results of this study are similar to various studies conducted in the other developing countries; as far as most frequent culprit lesion is concerned. The difference between this study and other studies done in African countries was more percentage $(77.5 \%)$ of the patients with esophageal varices as compared to African population (40.6\%). Another difference is in the gender distribution of esophageal varices as compared to other African studies where male to female ratio of approximately $2: 1.7,910,11$ These findings are in contrast to the findings in most of the studies conducted in western population in which peptic ulcer was the top most cause of bleeding from upper gastrointestinal tract.5, $5,8,9$

These differences may be due to high prevalence of chronic hepatitis and cirrhosis of liver in our 
population. Hepatitis B is the cause of acute viral hepatitis in approximately one third of cases. ${ }^{12}$ The prevalence of $\mathrm{HCV}$ infection in our population was found to be $4-12.5 \% .^{13}$ as compared to the developed countries like USA where the prevalence of $\mathrm{HCV}$ infection $1.6 \%{ }^{14}$ and the prevalence of $\mathrm{HBV}$ is $0.2 \%{ }^{15}$

The findings in this study are consistent with the findings of other local research done in our population in regard to the most frequent cause of bleeding from UGIT. ${ }^{16,17}$

Peptic ulcer is the common cause of UGIB in western population, in our study it is the second common cause with similar frequency as found in African population. (6.7\% in our study versus $6.2 \%$ in African studies). Peptic ulcer ranks third in causing bleeding from upper gastrointestinal tract in African patients. $3,10,18,19,20$

Other less common causes of UGIB found in our study were fundal varices $(5.8 \%)$, esophageal ulcer and esophagitis (2.5\%).

Limitations of this study are the small number of patients were studied, therefore cannot be generalized to the whole population but the findings in this study are similar to the other studies in regard to the most frequent etiology of UGIB done in our population with large number of patients.

\section{CONCLUSION}

Esophageal varices are the most common cause of upper gastrointestinal bleeding in our population due to the high prevalence of hepatitis infection and chronic liver disease in our population This study and other studies done in Pakistan shows that esophageal varices is alarming problem in our population presented with upper gastrointestinal bleeding and it is important to note that esophageal varices causes significant morbidity and mortality. It results from the complication of portal hypertension mostly due to decompensated liver cirrhosis by viral hepatitis in our circumstances, therefore prevention of chronic hepatitis and cirrhosis is vital, because once esophageal varices has developed the prognosis is dismal.

Copyright (C) 20 Jan, 2017.

\section{REFERENCES}

1. Gralnek IM, Jensen MD, Gornbein J, et al. Clinical and economic outcomes of patients with severe peptic ulcer hemorrhage and non bleeding visible vessels: An analysis of two prospective clinical trials. Am J Gastrointerol. 1998; 93:2047-2056.

2. Fallah MA, Prakash C, Edmundowicz S. Acute gastrointestinal bleeding. Med Clin North Am. 2000 Sep; 84(5):1183-1208.

3. Jutabha R, Jensen MD. Management of upper gastrointestinal bleeding in patients with chronic liver disease. Med Clin North Am. 1996; 80(5):10351068.

4. Baradarian R, Ramdhaney S, Chapalamadugu R, Skoczylas L, Wang K, Rivilis S, et al. Early intensive resuscitation of patients with upper gastrointestinal bleeding decreases mortality. Am J Gastroenterol. 2004 Apr; 99(4):619-622.

5. Yachimski PS, Friedman LS. Gastrointestinal bleeding in the elderly. Nat Clin Pract Gastroenterol Hepatol. 2008; 5(2):80-93.

6. Adang RP, Vismans JF, Talmon JL, et al. Appropriateness of. A14-A14. indications for diagnostic upper gastrointestinal endoscopy: Association with relevant endoscopic diseases. Gastroint Endosc. 1995; 42(5):390-397.

7. Van Leerdam ME. Epidermiology of acute uppergastrointestinal bleeding: Best practice research. clinical gastroenterology. 2008; 22:209-224.

8. Mwanahawa S, Segni $M$, Charles $M$, et al. The etiology, management and clinical outcome of upper gastrointestinal bleeding among patients admitted at the Kilimanjaro Christian Medical Centre in Moshi, Tanzania. Tanzania journal of Health Research. 2010; 12(4):304-308.

9. Cook DJ, Guyatt GH, Salena BJ, Laine LA. Endoscopic therapy for non- variceal upper gastrointestinal haemorrhage: a metaanalysis. Gastroenterology. 1992; 102:139-148.

10. Malu AO, Wali SS, Kazmi R, Macaulay D, Fakunlay YM. Upper GI endoscopy in Zaria, Northern Nigeria. West Afr J Med. 1990; 9:279-284.

11. Pruthi HS, Sharma SK, Singh B, Anand AC. Aetiology of upper gastrointestinal bleeding: an endoscopic 
study. MJAFI. 2000; 56:188-191.

12. Tanwani AK, Ahmad N. Prevalence of Hepatitis B surface antigen and anti Hepatitis $C$ antibody in laboratory based data at Islamabad. J Surg 2000; 19:25-9.

13. Hashim R, Hussain AB, Rehman K. Seroprevalence of Hepatitis-C virus antibodies among healthy young men in Pakistan. Pak J Med Res 2005; 44:140-2.

14. Armstrong GL, Wasley A, Simard EP, McQuillan GM, Kuhnert WL, Alter MJ. The prevalence of hepatitis C virus infection in the United States, 1999 through 2002. Ann Intern Med 2006; 144:770-771.

15. Kim WR, Ishitani MB, Dickson ER. Rising burden of hepatitis B in the United States: Should the other virus be forgotten. Hepatology 2002; 36:222.

16. Adam T, Javid F, Khan S. Upper Gastrointestinal bleeding: An etiological study of 552 cases. J Pak
Inst Med Sci 2004; 15:845-48.

17. Chaudhary AW, Tabassum HM, Chaudhary MA. Pattern of upper gastrointestinal bleeding at Rahim Yar Khan. Ann K E Med Coll 2005; 11(3):282-3.

18. Rockall TA, Logan RF, Devlin HB, Northfield TC. Incidence and mortality from acute upper gastrointestinal haemorrhage in the United Kingdom. Br Med J. 1995; 311:222-6.

19. Gostout CJ, Wang KK, Ahlquist DA, Clain JE, Hughes RW, Larson MV, Petersen BT, Shroeder KW, Tremaine WJ, Vigianno TR. Acute gastrointestinal bleeding. Experience of a specialised management team. $J$ Clin Gastroenterol. 1992; 14:260-67.

20. Morgan AG, MacAdam WA, Walmsley GL, Jessop A, Horrocks JC, de Dombal FT. Clinicalfindings, early endoscopy and multivariate analysis in patients bleeding from the upper gastrointestinal tract. $\mathrm{Br}$ Med J.1985; 291(6495):237-40.

\section{PREVIOUS RELATED STUDY}

Javed Iqbal. UPPER GASTROINTESTINAL BLEEDING; ASSESSMENT OF CAUSES AND COMPARISON WITH OTHER RELEVANT STUDIES (Original) Prof Med Jour 11(4) 406-410 Oct, Nov, Dec, 2004.

\section{AUTHORSHIP AND CONTRIBUTION DECLARATION}

\begin{tabular}{|c|l|l|l|}
\hline Sr. \# & \multicolumn{1}{|c|}{ Author-s Full Name } & \multicolumn{1}{|c|}{ Contribution to the paper } & Author=s Signature \\
\hline 1 & Dr. Mughees Ather & Data Collection & \\
2 & Dr. Muhammad Sarfraz & $\begin{array}{l}\text { Results with references \& } \\
\text { Discussion } \\
\text { Discussion \& Result }\end{array}$ \\
\hline 3 & Dr. Muhammad Zakria & Diss
\end{tabular}

